\title{
Periodontitis and pulmonary function: a Mendelian randomization study
}

\author{
Sebastian-Edgar Baumeister ${ }^{1}\left(\mathbb{D} \cdot\right.$ Michael Nolde $^{1,2} \cdot$ Birte Holtfreter $^{3} \cdot$ Hansjörg Baurecht $^{4} \cdot$ Sven Gläser $^{5,6}$. \\ Thomas Kocher $^{3}$ - Benjamin Ehmke ${ }^{7}$
}

Received: 12 April 2021 / Accepted: 19 May 2021 / Published online: 27 May 2021

(c) The Author(s) 2021

\begin{abstract}
Objectives Observational research suggests that periodontitis affects pulmonary function; however, observational studies are subject to confounding and reverse causation, making causal inference and the direction of these associations difficult. We used Mendelian randomization (MR) to assess the potential causal association between genetic liability to periodontitis and pulmonary function.

Materials and methods We used six single-nucleotide polymorphisms (SNPs) associated with periodontitis $\left(P<5 \times 10^{-6}\right)$ from a genome-wide association study (GWAS) of 17,353 European descent periodontitis cases and 28,210 controls from the GeneLifestyle Interactions in Dental Endpoints consortium and the UK Biobank, and related these to SNPs from a lung function GWAS including 79,055 study participants of the SpiroMeta Consortium.

Results MR analysis suggested no effect of periodontitis on the ratio of forced expiratory volume in one second to lower forced vital capacity (standard deviation increment in outcome per doubling of the odds of the exposure (95\% confidence interval $)=-0.004(-0.028 ; 0.020))$. Replication analysis using genetic instruments from two different GWAS and sensitivity analyses to address potential pleiotropy led to no substantial changes in estimates.

Conclusions Collectively, these findings do not support a relationship between genetic liability for periodontitis and pulmonary function.
\end{abstract}

Clinical relevance Periodontitis does not seem to be a risk factor for worsening of pulmonary function.

Keywords Periodontitis $\cdot$ Lung function $\cdot$ Mendelian randomization

Periodontitis is a chronic, bacteria-initiated inflammatory disease, affecting $50 \%$ of adults, with $11.2 \%$ suffering from severe periodontitis [1]. Chronic obstructive pulmonary disease (COPD) is a disease with a growing global burden. It is characterized by persistent symptoms and progressive airflow limitations diagnosed by lung function testing. As

Sebastian-Edgar Baumeister

sebastian.baumeister@uni-muenster.de

1 Institute of Health Services Research in Dentistry, University of Münster, Albert-Schweitzer-Campus 148149 , Münster, Germany

2 Chair of Epidemiology, University of Augsburg, Augsburg, Germany

3 Unit of Periodontology, Department of Restorative Dentistry, Periodontology, Endodontology, and Preventive and Pediatric Dentistry, University Medicine Greifswald, Greifswald, Germany
COPD and periodontitis are both characterized by neutrophilic inflammation with subsequent proteolytic destruction of connective tissue, it has been proposed that they share common pathophysiological processes. Periodontitis has been linked to a higher risk of COPD and worsening of pulmonary function in previous observational research $[2,3]$.
4 Department of Epidemiology and Preventive Medicine, University of Regensburg, Regensburg, Germany

5 Department of Internal Medicine B Cardiology, Intensive Care, Pulmonary Medicine and Infectious Diseases, University Medicine Greifswald, Greifswald, Germany

6 Vivantes Klinikum Neukölln Und Spandau, Klinik Für Innere Medizin - Pneumologie Und Infektiologie, Berlin, Germany

7 Clinic for Periodontology and Conservative Dentistry, University of Münster, Münster, Germany 
The latest systematic review identified seven cross-sectional and prospective studies and reported a pooled, confounderadjusted odds ratio of 1.78 (95\% confidence interval (CI): $1.04 ; 3.05)$ for COPD [2]. Proposed pathways by which periodontitis might influence pulmonary function are by mechanical aspiration of oral contents into the respiratory tree, the overspill of locally produced inflammatory mediators into the systemic circulation or oral or lung-derived bacteremia activating an acute-phase response, and reactive oxygen species and cytokine release by systemic neutrophils at distant sites $[4,5]$.

Yet, the observed association between periodontitis and pulmonary function could also be due to confounding, such as tobacco smoking [5], which is difficult to control when relying on observational study designs. One approach to account for observational bias and strengthen causal inference is the method of Mendelian randomization (MR), a form of instrumental variable analysis [6]. The MR method diminishes confounding by environmental factors because alleles are randomly allocated when passed from parents to offspring at conception and avoids reverse causation because disease cannot affect genotype. In MR, the instrument comprises one or more genetic variants that are robustly associated with the exposure of interest. The most widely adopted approach is to rely on inferences from single-nucleotide polymorphisms (SNPs) identified through genome-wide association studies (GWAS).

\section{Methods}

We performed two-sample MR analysis using summary statistics from the largest available GWAS on periodontitis and pulmonary function $[7,8]$. We used 6 SNPs associated with periodontitis $\left(P<5 \times 10^{-6}\right)$, after accounting for linkage disequilibrium $\left(\mathrm{r}^{2}=0.001\right.$, window size $\left.=10 \mathrm{mB}\right)$, as instruments from GWAS of 17,353 European decent periodontitis cases and 28,210 controls from the GeneLifestyle Interactions in Dental Endpoints consortium and UK Biobank [8], and related these to SNP-lung function associations from GWAS of 79,055 European decent study participants of the
SpiroMeta Consortium [7]. After data harmonization, we calculated Wald ratios by dividing the linear regression coefficients for forced expiratory volume in one second $\left(\mathrm{FEV}_{1}\right)$, lower forced vital capacity (FVC), and $\mathrm{FEV}_{1} / \mathrm{FVC}$ ratio by the corresponding log OR of the same SNP in the GWAS for periodontitis; and obtained standard errors by the delta method. Wald ratios were pooled using the primary multiplicative random effects inverse-variance-weighted (IVW) analyses [9]. We transformed the effect estimates of the binary exposure to reflect doubling in the odds of the exposure on the per one standard deviation (1-SD) increment of the outcome [10]. MR rests on the assumptions that the instrument is robustly associated with the exposure (I), is not associated with confounders (II), and is not associated with the outcome other than via its association with the exposure (III). If the instrument-exposure association is weak, this might reduce the plausibility of assumption I. Violations of assumptions II and III can occur through horizontal pleiotropy, whereby the instruments exert an effect on the outcome independent of the exposure. We used more liberal SNP selection thresholds $\left(P<5 \times 10^{-4}, P<5 \times 10^{-5}\right)$ to strengthen the instrument and applied weak instrument analyses $[6,9,11]$. We investigated pleiotropy by searching for previously reported associations of instruments with tobacco smoking in PhenoScanner (www. phenoscanner.medschl.cam.ac.uk/) and the GWAS Catalog (www.ebi.ac.uk/gwas/); investigated the Cochran Q heterogeneity test, the $\mathrm{I}^{2}$ statistic, and the MR-Egger intercept test; performed leave-one-SNP-out analysis; and applied a suite of pleiotropy-robust methods (weighted median, robust adjusted profile score, radial regression, MR-pleiotropy residual sum and outlier) [6, 12]. We used two additional GWAS [13, 14] of aggressive and chronic periodontitis to identify instruments in replication analyses and adopted a negative control outcome approach [15]. Height at age 10 years served as a negative control. SNP-outcome associations for height at age 10 years were taken from the Neale 2017 UK Biobank phenome-wide GWAS included in mrbase.org (access date 2021 February 11). As the periodontitis age of onset is typically after puberty, if our instruments affect lung function solely through periodontitis phenotypes, we expect to find no effect on prepuberty height.
Lung Function

Periodontitis
FEV1
FVC
FEV1/FVC

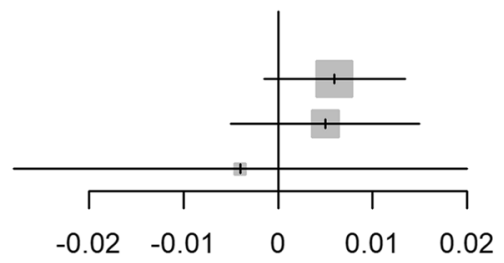

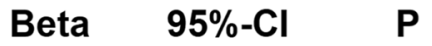

\author{
$0.006 \quad(-0.001 ; 0.014) \quad 0.077$ \\ $0.005 \quad(-0.005 ; 0.015) \quad 0.362$ \\ $\begin{array}{lll}-0.004 & (-0.028 ; 0.020) & 0.739\end{array}$
}

Fig. 1 Per one standard deviation change (beta) in forced expiratory volume in $1 \mathrm{~s}$ (FEV1), forced vital capacity (FVC), and FEV1/FVC ratio associated with doubling in the prevalence of genetic liability to periodontitis using the inverse-variance-weighted method. $C I$, confidence interval; $P, P$ value 


\section{Results}

The IVW analysis did not suggest an effect of genetic liability to periodontitis on $\mathrm{FEV}_{1}, \mathrm{FVC}$, and $\mathrm{FEV}_{1} / \mathrm{FVC}$ (Fig. 1). For example, a 1-SD of the $\mathrm{FEV}_{1} / \mathrm{FVC}$ ratio decreased by -0.004 (95\% CI: $-0.028 ; 0.020)$ per doubling in the odds of periodontitis. The primary analyses were supported when using more liberal SNP selection thresholds and in weak instrument analyses. We found no previously reported associations of genetic variants with tobacco smoking. The IVW estimates were consistent with estimates from robust methods. There was minimal heterogeneity between Wald ratios, and the MR-Egger intercept analyses did not indicate directional pleiotropy. IVW leaveone-out analysis did not identify any leverage points with high influence. In replication analysis, when summary data from two different GWAS of periodontitis were used, effect sizes were similar to the primary analysis. In negative control outcome analysis, the association (IVW Beta $=0.009$, $95 \%$ CI: $-0.001 ; 0.003$ ) with height at 10 years provided additional reassurance for the robustness of the primary analysis.

\section{Discussion}

The association between periodontitis and pulmonary function seen in previous observational studies was not replicated when applying MR and could have been due to unobserved environmental confounding or selection bias [5]. The study had limitations. The phenotypic variance explained by the 6 SNPs in primary analysis was only $0.8 \%$; however, the minimum F statistic was 22 and did not raise concerns of weak instrument bias. Additional weak instrument analyses provided higher statistical power and also did not suggest an association. The biologic mechanisms of the selected SNPs are unknown; however, sensitivity analyses failed to find evidence for horizontal pleiotropy. The primary periodontitis GWAS [8] used a broadly defined phenotype including the Centers for Disease Control and Prevention/American Academy of Periodontology definition of periodontitis or participant-reported diagnosis of periodontitis, which might have introduced measurement error. However, classical measurement error in the exposure does not affect asymptotic estimates from instrumental variable analysis. Also, replication analysis using SNPs from two GWAS [13, 14] of clinically defined periodontitis yielded similar point estimates. Furthermore, the phenotype was binary so we could not assess potential dose-dependent changes in pulmonary function. Finally, the SNP effect estimates were obtained from European studies, and caution is warranted before generalizing findings to other populations.

Collectively, our data provided preliminary evidence that genetically proxied long-term exposure to periodontitis does not worsen pulmonary function. We emphasize the importance of triangulating multiple lines of experimental and observational research to strengthen causal inference [16].

Acknowledgements The authors acknowledge and thank the investigators of the primary GWAS for making the summary data publicly available.

Funding Open Access funding enabled and organized by Projekt DEAL.

\section{Declarations}

Conflict of interest The authors declare no competing interests.

Open Access This article is licensed under a Creative Commons Attribution 4.0 International License, which permits use, sharing, adaptation, distribution and reproduction in any medium or format, as long as you give appropriate credit to the original author(s) and the source, provide a link to the Creative Commons licence, and indicate if changes were made. The images or other third party material in this article are included in the article's Creative Commons licence, unless indicated otherwise in a credit line to the material. If material is not included in the article's Creative Commons licence and your intended use is not permitted by statutory regulation or exceeds the permitted use, you will need to obtain permission directly from the copyright holder. To view a copy of this licence, visit http://creativecommons.org/licenses/by/4.0/.

\section{References}

1. Peres MA, Macpherson LMD, Weyant RJ, Daly B, Venturelli R, Mathur MR et al (2019) Oral diseases: a global public health challenge. Lancet 394(10194):249-260

2. Gomes-Filho IS, Cruz SSD, Trindade SC, Passos-Soares JS, Carvalho-Filho PC, Figueiredo A et al (2020) Periodontitis and respiratory diseases: a systematic review with meta-analysis. Oral Dis 26(2):439-446

3. Heinrich J, Thiering E, Jörres RA, Schulz H, Kühnisch J, Standl M (2019) Lung function and oral health in adolescents. Eur Respir J 53(3):1801951

4. Hobbins S, Chapple IL, Sapey E, Stockley RA (2017) Is periodontitis a comorbidity of COPD or can associations be explained by shared risk factors/behaviors? Int J Chron Obstruct Pulmon Dis 12:1339-1349

5. Tan L, Wang H, Pan C, Zhao J (2016) Periodontal health and chronic obstructive pulmonary disease stratified by smoking: a meta-analysis. Int J Clin Exp Med 9(12):23190-23197

6. Burgess S, Smith GD, Davies NM, Dudbridge F, Gill D, Glymour $\mathrm{MM}$ et al (2020) Guidelines for performing Mendelian randomization investigations. Wellcome Open Research 4(186): 186 
7. Shrine N, Guyatt AL, Erzurumluoglu AM, Jackson VE, Hobbs BD, Melbourne CA et al (2019) New genetic signals for lung function highlight pathways and chronic obstructive pulmonary disease associations across multiple ancestries. Nat Genet 51(3):481-493

8. Shungin D, Haworth S, Divaris K, Agler CS, Kamatani Y, Keun Lee M et al (2019) Genome-wide analysis of dental caries and periodontitis combining clinical and self-reported data. Nat Commun 10(1):2773

9. Burgess S, Dudbridge F, Thompson SG (2016) Combining information on multiple instrumental variables in Mendelian randomization: comparison of allele score and summarized data methods. Stat Med 35(11):1880-1906

10. Burgess S, Labrecque JA (2018) Mendelian randomization with a binary exposure variable: interpretation and presentation of causal estimates. Eur J Epidemiol 33(10):947-952

11. Morrison J, Knoblauch N, Marcus JH, Stephens M, He X (2020) Mendelian randomization accounting for correlated and uncorrelated pleiotropic effects using genome-wide summary statistics. Nat Genet 52(7):740-747

12. Slob EA, Burgess S (2020) A comparison of robust Mendelian randomization methods using summary data. Genet Epidemiol 20:1-17

13. Offenbacher S, Divaris K, Barros SP, Moss KL, Marchesan JT, Morelli T et al (2016) Genome-wide association study of biologically informed periodontal complex traits offers novel insights into the genetic basis of periodontal disease. Hum Mol Genet 25(10):2113-2129

14. Munz M, Richter GM, Loos BG, Jepsen S, Divaris K, Offenbacher S et al (2019) Meta-analysis of genome-wide association studies of aggressive and chronic periodontitis identifies two novel risk loci. Eur J Hum Genet 27(1):102-113

15. Sanderson E, Richardson TG, Hemani G, Davey Smith G (2021) The use of negative control outcomes in Mendelian randomization to detect potential population stratification. Int J Epidemiol. https://doi.org/10.1093/ije/dyaa288

16. Munafò MR, Higgins JPT, Smith GD (2020) Triangulating evidence through the inclusion of genetically informed designs. Cold Spring Harb Perspect Med. https://doi.org/10.1101/cshperspect. a040659

Publisher's note Springer Nature remains neutral with regard to jurisdictional claims in published maps and institutional affiliations. 